\title{
Characterizing Entanglement Entropy Produced by Non-Linear Scalar Interactions During Inflation
}

\author{
Dan Mazur and Jeremy S. Heyl* \\ Department of Physics and Astronomy, University of British Columbia \\ 6224 Agricultural Road, Vancouver, British Columbia, Canada, V6T 1 Z1
}

(Dated: October 31, 2018)

\begin{abstract}
The density fluctuations that we observe in the universe today are thought to originate from quantum fluctuations produced during a phase of the early universe called inflation. By evolving a wavefunction describing two coupled Fourier modes of a scalar field forward through an inflationary epoch, we demonstrate that non-linear effects can result in a generation of entanglement entropy between modes with different momenta in a scalar field during the inflationary period when just one of the modes is observed. Through this mechanism, the field would experience decoherence and appear more like a classical distribution today; however the mechanism is not sufficiently efficient to explain classicality. We find that the amount of entanglement entropy generated scales roughly as a power law $S \propto \lambda^{1.75}$, where $\lambda$ is the coupling coefficient of the non-linear potential term. We also investigate how the entanglement entropy scales with the duration of inflation and compare various entanglement measures from the literature with the von Neumann entropy. This demonstration explicitly follows particle creation and interactions between modes; consequently, the mechanism contributing to the generation of the von Neumann entropy can be easily seen.
\end{abstract}

PACS numbers: 98.80.Cq, 98.80.Qc

\section{INTRODUCTION}

Most modern cosmological models include a period in the universe's history called inflation during which the scale parameter increased exponentially with the proper time of a comoving observer. This period was originally introduced to address the horizon and flatness problems of cosmology 1]. More recently, however, research on inflation has been toward understanding structure formation [2, 3, 4]. The distribution of galaxies and clusters that we observe in the universe today are thought to have originated from fluctuations of a quantized field created during inflation [5, [6]. A thorough review of structure formation and inflationary cosmology can be found in Liddle and Lyth [7].

Despite their quantum mechanical origins, the latetime evolution of these fluctuations is treated in a classical framework. It is therefore important to understand the quantum-to-classical transition made by these fluctuations (for a recent review, see [8]). The classicality of a quantum system is often discussed in the context of decoherence. That is, as a quantum system interacts with unobserved environmental influence, that system loses quantum coherence and begins to behave as a classical statistical distribution.

The quantized field may of course be the inflaton itself, which drives the inflation of the universe, or it could be another quantized field that produces density fluctuations as in curvaton models or the gravitational field. It is possible, in principle, that non-classical correlations from an inflationary period in our universe's history may one

\footnotetext{
${ }^{*}$ Canada Research Chair
}

day be observed. But this depends on the decoherence that the scalar or tensor field has experienced since the beginning of inflation. Several authors have investigated decoherence of the density fluctuations by calculating the entropy of cosmological perturbations created during inflation 9, 10, 11, 12, 13, 14].

It has been suggested [15] that decoherence is unlikely to occur during inflation because the Bunch-Davies state occupied by the scalar field during inflation is similar to the Minkowski vacuum. Because the ordinary Minkowski vacuum does not decohere, we would not expect to see any decoherence from a scalar field during inflation. In the particle-based picture adopted for the present analysis, it becomes clear that the scalar field does undergo decoherence when the potential is non-linear.

Since decoherence is a necessary condition for the emergence of classicality in a quantum system [16], nonlinearities in the scalar field help to explain the classical matter distribution that we observe today. This simple model demonstrates that this entropy generation can occur during inflation itself and does not depend on the reheating process at the end of inflation; therefore, the results are perhaps most interesting for cosmological scalar fields that do not participate in reheating. For such fields, the non-linear interactions do not generate a sufficient amount of decoherence to result in classicality for the fields.

Here, we examine the case where certain modes of a field play the role of the environmental influence and cause decoherence when a non-linearity in the potential allows the modes to interact 15, 17, 18. We discuss a simulation that was performed to compute the entanglement entropy between such modes in a very transparent model that follows particle creation and the interaction between modes during the inflationary period. The en- 
tropy is computed as inflation progresses to demonstrate the decoherence of a scalar field.

Computing the entanglement entropy of a large quantum system is a computationally difficult task since it involves diagonalizing the density matrix. To evaluate several possible expediencies, we have compared our results to other measures of entanglement and correlations between modes. We have found that the other measures considered share a similar qualitative behaviour with the entanglement entropy and can be much easier to compute. Therefore, for some applications, these measures may be useful as stand-in quantities in simulations where the entanglement entropy is too costly to compute. We verify several efficient methods to characterize the entropy.

\section{COSMOLOGICAL SCALAR-FIELD EVOLUTION}

We would like to investigate the evolution of a scalar field in an isotropic, homogeneous, flat spacetime. The analysis for this situation is covered extensively in part I, chapter 6 of Mukhanov et al. [19]. The relevant metric for this evolution is

$$
d s^{2}=a^{2}(\tau)\left(d \tau^{2}-d \mathbf{x}^{2}\right)
$$

where $\tau$ is the conformal time, which is related to the comoving time by $d t=a(\tau) d \tau$, and $\mathbf{x}$ is a comoving displacement. For simplicity we will take $a(\tau)=-(H \tau)^{-1}$ (pure deSitter expansion) during inflation.

The evolution of a scalar field $\phi$ is governed by its Lasion containing up to first derivatives is

$$
\mathcal{L}=\frac{1}{2} g^{\mu \nu} \partial_{\mu} \phi \partial_{\nu} \phi-V(\phi) .
$$

For simplicity we will neglect the mass of the scalar field in the potential that couples the Fourier modes of the grangian $\mathcal{L}$. The lowest-order Lorentz-invariant expresduring inflation $(m \ll H)$. We include a non-linearity

field. Even if the field itself is free, its self-gravity will introduce an interaction potential of the form [15, 18]

$$
V=\lambda M_{\mathrm{Pl}} \phi^{3}
$$

Although the $\phi^{3}$ potential is generally unstable, one should interpret this as an effective potential to account for the gravitational self-interaction, so the instability is not surprising because the gravitational self-interaction is generally unstable.

\section{A. Mode coupling during inflation}

For this analysis, we choose to use a simple model in which the universe contains only particles with four possible momenta: $\pm \mathbf{k}$ and $\pm 2 \mathbf{k}$. Given this requirement, we construct a Hamiltonian which incorporates a coupling term between these two Fourier modes so that we can observe the effect this non-linearity has on the entanglement between modes during inflation.

The creation and annihilation operators satisfy the following commutation relations

$$
\begin{aligned}
& {\left[a_{\mathbf{k}}, a_{\mathbf{k}^{\prime}}^{\dagger}\right]=\delta^{(3)}\left(\mathbf{k}-\mathbf{k}^{\prime}\right)} \\
& {\left[a_{\mathbf{k}}^{\dagger}, a_{\mathbf{k}^{\prime}}^{\dagger}\right]=\left[a_{\mathbf{k}}, a_{\mathbf{k}^{\prime}}\right]=0 .}
\end{aligned}
$$

Including our potential term (3), the action for the field is

$$
S=\frac{1}{2} \int d^{4} x \sqrt{-g}\left[\partial_{\mu} \phi \partial^{\mu} \phi+\lambda M_{\mathrm{Pl}} \phi^{3}\right]
$$

Following the steps outlined by ref. [20], we arrive at the following expression for the action.

$$
S=\frac{1}{2} \int d^{4} x a^{2}\left\{\left[\frac{\partial \phi}{\partial \tau}\right]^{2}-\left[\nabla \phi^{2}\right]+a^{2} \lambda M_{\mathrm{Pl}} \phi^{3}\right\}
$$

If we make the substitution $u=a \phi=\frac{1}{(2 \pi)^{3 / 2}} \int d^{3} k u_{k}(\tau) e^{i \mathbf{k} \cdot \mathbf{r}}$, the action becomes

$$
S=\frac{1}{2} \int d \tau d^{3} k\left[\left|\frac{\partial u_{k}}{\partial \tau}\right|^{2}-\left(k^{2}+m_{\mathrm{eff}}^{2}\right)\left|u_{k}\right|^{2}-\frac{\lambda M_{\mathrm{Pl}}}{(2 \pi)^{1 / 2} a} \int d^{3} k^{\prime} d^{3} k^{\prime \prime} u_{\mathbf{k}} u_{\mathbf{k}^{\prime}} u_{\mathbf{k}^{\prime \prime}} \delta^{(3)}\left(\mathbf{k}+\mathbf{k}^{\prime}+\mathbf{k}^{\prime \prime}\right)\right]
$$

where the effective mass is $m_{\mathrm{eff}}^{2}=-2 \frac{Q}{\tau^{2}}$,

$$
Q \equiv \frac{1}{(1+3 w)^{2}}[(1-3 w)]
$$

and $w=p / \rho$ is the equation of state parameter.

The Hamiltonian is, then,

$$
H=\frac{1}{2} \int d^{3} k\left[\left|\frac{\partial u_{\mathbf{k}}}{\partial \tau}\right|^{2}+\left(k^{2}+m_{\mathrm{eff}}^{2}\right)\left|u_{\mathbf{k}}\right|^{2}+\frac{\lambda M_{\mathrm{Pl}}}{\sqrt{2 \pi} a} \int d^{3} k^{\prime} u_{\mathbf{k}} u_{\mathbf{k}^{\prime}} u_{-\left(\mathbf{k}+\mathbf{k}^{\prime}\right)}\right] .
$$


In general, we have $u_{\mathbf{k}}=g(k, \tau) a_{\mathbf{k}}+g^{*}(k, \tau) a_{-\mathbf{k}}^{\dagger}$. Putting this into to the Hamiltonian, (10), and neglecting terms that do not conserve energy in flat spacetime gives

$$
\begin{aligned}
H & =\frac{1}{2} \int d^{3} k\left[\left(\left|\frac{\partial g(k, \tau)}{\partial \tau}\right|^{2}+|g(k, \tau)|^{2}\left(k^{2}+m_{\mathrm{eff}}^{2}\right)\right)\left(a_{\mathbf{k}}^{\dagger} a_{\mathbf{k}}+a_{\mathbf{k}} a_{\mathbf{k}}^{\dagger}\right)\right. \\
& +\left(\frac{\partial g^{* 2}(k, \tau)}{\partial \tau}+g^{* 2}(k, \tau)\left(k^{2}+m_{\mathrm{eff}}^{2}\right)\right) a_{-\mathbf{k}}^{\dagger} a_{\mathbf{k}}^{\dagger}+\left(\frac{\partial g^{2}(k, \tau)}{\partial \tau}+g^{2}(k, \tau)\left(k^{2}+m_{\mathrm{eff}}^{2}\right)\right) a_{\mathbf{k}} a_{-\mathbf{k}} \\
& \left.+\frac{\lambda M_{\mathrm{Pl}}}{\sqrt{2 \pi} a}\left[g(k, \tau) g(k, \tau) g^{*}(2 k, \tau) a_{\mathbf{2} \mathbf{k}}^{\dagger} a_{\mathbf{k}} a_{\mathbf{k}}+g^{*}(k, \tau) g^{*}(k, \tau) g(2 k, \tau) a_{\mathbf{2 k}} a_{\mathbf{k}}^{\dagger} a_{\mathbf{k}}^{\dagger}\right]\right]
\end{aligned}
$$

The mode function is normally chosen to be

$$
g(k, \tau)=-\frac{1}{\sqrt{2 k^{3}}}(i-k \tau) \frac{e^{-i k \tau}}{\tau}
$$

as this choice satisfies the equation of motion for the free field during a deSitter phase and because it simplifies the Hamiltonian to one that commutes with the number operator since, when $Q=1$,

$$
\frac{\partial g^{2}(k, \tau)}{\partial \tau}+g^{2}(k, \tau)\left(k^{2}+m_{\text {eff }}^{2}\right)=0 .
$$

However, this choice is not practical for our calculation because the scalar field is not free; therefore, this choice does not satisfy the field equation of motion, and in fact it complicates the Hamiltonian because, for example

$$
g(k, \tau) g(k, \tau) g^{*}(2 k, \tau)=\frac{1}{4 k^{9 / 2} \tau}\left(2(k \tau)^{3}-3 i(k \tau)^{2}-i\right)
$$

does not have a simple dependence on $\tau$ and the simplifications provided by (12) are lost.

We would like to know the amount of entropy at the end of inflation during radiation domination. The usual way to proceed is to select the mode function (12) and use this to determine the equation of motion for the scalar field during inflation. We would then determine the Bogoliubov coefficients at the transition from inflation to radiation domination. After performing the transformation, we would compute the amount of entropy from the transformed density matrix.

However, we can simplify the problem by instead choosing a mode function that describes the system during radiation domination and use this mode function to compute the entire evolution. The choice of function $g(k, \tau)$ is flexible due to the vacuum ambiguity and is related to choosing the set of states that the creation and annihilation operators act upon. Any choice will provide us with a complete basis with which we can describe any state of the field. The arrbitrariness of the mode function is also discussed in [21].

For us, it is most prudent to choose the simple function

$$
g(k, \tau)=\frac{1}{\sqrt{2 k}} e^{-i k \tau}
$$

which defines the vacuum both during radiation domination and for scales much smaller than the horizon even during the de Sitter phase. Thus, we can make a very natural connection between our initial state and our final state. The choice is as arbitrary as choosing to perform a calculation in classical mechanics in a rotating frame rather than an inertial frame.

Correctly interpreting the wavefunction where (15) is inappropriate (i.e. after horizon exit during a de Sitter phase) would require a Bogoliubov transformation, but for our purposes we do not require this. We are only interested in calculating the entropy after the transition to radiation domination where our choice of mode function corresponds to the usual creation and annihilation operators for this background. Therefore, we avoid transformations entirely since we already have the required description of our wavefunction.

With the choice (15), the Hamiltonian is not constant in time even without the non-linear couplings. In particular the mass depends on time; this choice is similar in spirit to the calculations of Guth and $\mathrm{Pi}([2])$. Heyl [20] has shown that for a free scalar field that this choice gives the same results as the standard function $g(k, \tau)$ and we refer the reader to that article for a more thorough discussion of the technique.

Choosing to use (15), we have

$$
u_{\mathbf{k}}=\frac{1}{\sqrt{2 k}}\left(e^{-i k \tau} a_{\mathbf{k}}+e^{i k \tau} a_{-\mathbf{k}}^{\dagger}\right)
$$


The nonlinear terms in the Hamiltonian provide a coupling mechanism between the modes of interest. To perform the integral over $d^{3} k^{\prime}$ in (10), we neglect the effect of the coupling on the modes that are not considered in our simulation and treat the functions $u_{\mathbf{k}}$ as constant on a spherical shell surrounding the momenta, $\mathbf{k}^{\prime}$, that we are interested in. For $u_{\mathbf{k}^{\prime}}=$ const. on spherical shells of constant volume around $\mathbf{k}$ and $2 \mathbf{k}$, the integral becomes

$$
\int d^{3} k^{\prime} u_{\mathbf{k}} u_{\mathbf{k}^{\prime}} u_{-\left(\mathbf{k}+\mathbf{k}^{\prime}\right)} \rightarrow V k^{3} u_{\mathbf{k}} u_{\mathbf{k}} u_{-2 \mathbf{k}}
$$

where $V=\frac{4}{3} \pi\left(\frac{4}{1+\sqrt[3]{2}}\right)^{3} \approx 23$ is a (somewhat arbitrary) geometrical constant.

Making this substitution, we arrive at the final form of the Hamiltonian.

$$
H=\int d^{3} \mathbf{k}\left[\left(k-\frac{Q}{\tau^{2} k}\right)\left(a_{\mathbf{k}}^{\dagger} a_{\mathbf{k}}+a_{\mathbf{k}} a_{\mathbf{k}}^{\dagger}\right)-\frac{Q}{\tau^{2} k}\left(a_{-\mathbf{k}} a_{\mathbf{k}} e^{-2 i k \tau}+a_{-\mathbf{k}}^{\dagger} a_{\mathbf{k}}^{\dagger} e^{2 i k \tau}\right)+\frac{\lambda V k^{3 / 2} M_{\mathrm{Pl}}}{4 \sqrt{2 \pi} a}\left(a_{\mathbf{2 k}}^{\dagger} a_{\mathbf{k}} a_{\mathbf{k}}+a_{\mathbf{2 k}} a_{\mathbf{k}}^{\dagger} a_{\mathbf{k}}^{\dagger}\right)\right]
$$

This Hamiltonian is similar to that used by ref. [20], generalized to allow for the interactions between Fourier modes.

Here, the two terms multiplied by the factor $\lambda$ are responsible for the annihilation of two particles from the $\mathbf{k}$ mode into a single particle from the $2 \mathbf{k}$ mode and the decay of an $2 \mathbf{k}$ mode particle into two $\mathbf{k}$ mode particles, respectively. As the two modes of the field exchange particles with each other, we expect that entanglement entropy will be generated in either of the modes observed individually.

We wish to use this Hamiltonian to evolve Fock space wavefunctions representing the number of particles in each of four modes: Those with $m^{+}$particles with momentum $2 \mathbf{k}^{\prime}, m^{-}$particles with momentum $-2 \mathbf{k}^{\prime}, n^{+}$particles with momentum $\mathbf{k}^{\prime}$, and $n^{-}$particles with momentum $-\mathbf{k}^{\prime}$.

$$
\begin{aligned}
|\psi\rangle= & \sum_{m^{+}=0}^{\infty} \sum_{m^{-}=0}^{\infty} \sum_{n^{+}=0}^{\infty} \sum_{n^{-}=0}^{\infty} B_{m^{+}, m^{-}, n^{+}, n^{-}}(\tau)\left(\frac{\left(a_{\mathbf{2} \mathbf{k}^{\prime}}^{\dagger} m^{+}\right.}{\sqrt{m^{+}}\left[\delta^{(3)}\left(2 \mathbf{k}^{\prime}-2 \mathbf{k}^{\prime}\right)\right]^{\frac{m^{+}}{2}}}\right)\left(\frac{\left(a_{-\mathbf{2} \mathbf{k}^{\prime}}^{\dagger}\right)^{m^{-}}}{\sqrt{m^{-}}\left[\delta^{(3)}\left(2 \mathbf{k}^{\prime}-2 \mathbf{k}^{\prime}\right)\right]^{\frac{m^{-}}{2}}}\right) \\
& \times\left(\frac{\left(a_{-\mathbf{k}^{\prime}}^{\dagger}\right)^{n^{-}}}{\sqrt{n^{+}}\left[\delta^{(3)}\left(\mathbf{k}^{\prime}-\mathbf{k}^{\prime}\right)\right]^{\frac{n^{+}}{2}}}\right)|0\rangle \\
= & \sum_{m^{+}, m^{-}, n^{+}, n^{-}=0}^{\infty} B_{m^{+}, m^{-}, n^{+}, n^{-}}(\tau)\left|m^{+}, 2 \mathbf{k}^{\prime}\right\rangle\left|m^{-},-2 \mathbf{k}^{\prime}\right\rangle\left|n^{+}, \mathbf{k}^{\prime}\right\rangle\left|n^{-},-\mathbf{k}^{\prime}\right\rangle .
\end{aligned}
$$

Whenever possible, we will use simplified notation such as

$$
|\psi\rangle=\sum_{n^{ \pm}, m^{ \pm}=0}^{\infty} B_{n^{ \pm}, m^{ \pm}}(\tau)\left|m^{ \pm}\right\rangle\left|n^{ \pm}\right\rangle
$$

In order to evolve the wavefunction forward in time, we replace $\tau$ with a new variable, $x=-1 /(k \tau)$. The equation of motion is then found from $i \frac{d}{d \tau}|\psi\rangle=H|\psi\rangle$, left multiplied by $\left\langle n^{ \pm}, \pm \mathbf{k}\right|\left\langle m^{ \pm}, \pm 2 \mathbf{k}\right|$. The following identities are needed to evaluate $H|\psi\rangle$ :

$$
\begin{aligned}
a_{\mathbf{k}}^{\dagger} a_{\mathbf{k}}\left|m^{ \pm}\right\rangle\left|n^{ \pm}\right\rangle= & {\left[m^{+} \delta^{(3)}\left(\mathbf{k}-2 \mathbf{k}^{\prime}\right)+n^{+} \delta^{(3)}\left(\mathbf{k}-\mathbf{k}^{\prime}\right)+m^{-} \delta^{(3)}\left(\mathbf{k}+2 \mathbf{k}^{\prime}\right)+n^{-} \delta^{(3)}\left(\mathbf{k}+\mathbf{k}^{\prime}\right)\right]\left|m^{ \pm}\right\rangle\left|n^{ \pm}\right\rangle } \\
\left(a_{\mathbf{k}}^{\dagger} a_{\mathbf{k}}+a_{\mathbf{k}} a_{\mathbf{k}}^{\dagger}\right)|\psi\rangle= & \left(2 a_{\mathbf{k}}^{\dagger} a_{\mathbf{k}}+Z\right)|\psi\rangle \\
a_{-\mathbf{k}} a_{\mathbf{k}}\left|m^{ \pm}\right\rangle\left|n^{ \pm}\right\rangle= & \sqrt{m^{+} m^{-}}\left(\delta^{(3)}\left(\mathbf{k}-2 \mathbf{k}^{\prime}\right)+\delta^{(3)}\left(\mathbf{k}+2 \mathbf{k}^{\prime}\right)\right)\left|m^{ \pm}-1\right\rangle\left|n^{ \pm}\right\rangle \\
& +\sqrt{n^{+} n^{-}}\left(\delta^{(3)}\left(\mathbf{k}-\mathbf{k}^{\prime}\right)+\delta^{(3)}\left(\mathbf{k}+\mathbf{k}^{\prime}\right)\right)\left|m^{ \pm}\right\rangle\left|n^{ \pm}-1\right\rangle \\
a_{-\mathbf{k}}^{\dagger} a_{\mathbf{k}}^{\dagger}\left|m^{ \pm}\right\rangle\left|n^{ \pm}\right\rangle= & \sqrt{\left(n^{+}+1\right)\left(n^{-}+1\right)}\left(\delta^{(3)}\left(\mathbf{k}-\mathbf{k}^{\prime}\right)+\delta^{(3)}\left(\mathbf{k}+\mathbf{k}^{\prime}\right)\right)\left|m^{ \pm}\right\rangle\left|n^{ \pm}+1\right\rangle \\
& +\sqrt{\left(m^{+}+1\right)\left(m^{-}+1\right)}\left(\delta^{(3)}\left(\mathbf{k}-2 \mathbf{k}^{\prime}\right)+\delta^{(3)}\left(\mathbf{k}+2 \mathbf{k}^{\prime}\right)\right)\left|m^{ \pm}+1\right\rangle\left|n^{ \pm}\right\rangle \\
a_{\mathbf{2} \mathbf{k}} a_{\mathbf{k}}^{\dagger} a_{\mathbf{k}}^{\dagger}\left|m^{ \pm}\right\rangle\left|n^{ \pm}\right\rangle= & \sqrt{m^{+}\left(n^{+}+1\right)\left(n^{+}+2\right)} \delta^{(3)}\left(2 \mathbf{k}-2 \mathbf{k}^{\prime}\right)\left|m^{+}-1\right\rangle\left|m^{-}\right\rangle\left|n^{+}+2\right\rangle\left|n^{-}\right\rangle \\
& +\sqrt{m^{-}\left(n^{-}+1\right)\left(n^{-}+2\right)} \delta^{(3)}\left(2 \mathbf{k}+2 \mathbf{k}^{\prime}\right)\left|m^{+}\right\rangle\left|m^{-}-1\right\rangle\left|n^{+}\right\rangle\left|n^{-}+2\right\rangle \\
a_{\mathbf{2} \mathbf{k}}^{\dagger} a_{\mathbf{k}} a_{\mathbf{k}}\left|m^{ \pm}\right\rangle\left|n^{ \pm}\right\rangle= & \sqrt{n^{+}\left(n^{+}-1\right)\left(m^{+}+1\right)} \delta^{(3)}\left(\mathbf{k}-\mathbf{k}^{\prime}\right)\left|m^{+}+1\right\rangle\left|m^{-}\right\rangle\left|n^{+}-2\right\rangle\left|n^{-}\right\rangle \\
& +\sqrt{n^{-}\left(n^{-}-1\right)\left(m^{-}+1\right)} \delta^{(3)}\left(\mathbf{k}+\mathbf{k}^{\prime}\right)\left|m^{+}\right\rangle\left|m^{-}+1\right\rangle\left|n^{+}\right\rangle\left|n^{-}-2\right\rangle
\end{aligned}
$$

where $Z=\left[a_{\mathbf{k}}, a_{\mathbf{k}}^{\dagger}\right]=\delta^{(3)}(\mathbf{k}-\mathbf{k})$ is an infinite constant. 
After some algebra, we find the time evolution of the states is given by

$$
\begin{aligned}
i \frac{d}{d x} A_{m^{ \pm}, n^{ \pm}}(x)= & -\frac{Q}{2}\left[\left(\sqrt{m^{+} m^{-}} A_{m^{ \pm}-1, n^{ \pm}}+\sqrt{n^{+} n^{-}} A_{m^{ \pm}, n^{ \pm}-1}\right) e^{-2 i \gamma / x}\right. \\
& \left.+\left(\sqrt{\left(n^{+}+1\right)\left(n^{-}+1\right)} A_{m^{ \pm}, n^{ \pm}+1}+\sqrt{\left(m^{+}+1\right)\left(m^{-}+1\right)} A_{m^{ \pm}+1, n^{ \pm}}\right) e^{2 i \gamma / x}\right] \\
& +\frac{\alpha}{x^{3}}\left[\left(\sqrt{\left(n^{+}-1\right)\left(n^{+}\right)\left(m^{+}+1\right)} A_{m^{+}+1, m^{-}, n^{+}-2, n^{-}}\right.\right. \\
& \left.+\sqrt{\left(n^{-}-1\right)\left(n^{-}\right)\left(m^{-}+1\right)} A_{m^{+}, m^{-}+1, n^{+}, n^{-}-2}\right) \\
& +\left(\sqrt{\left(m^{+}\right)\left(n^{+}+1\right)\left(n^{+}+2\right)} A_{m^{+}-1, m^{-}, n^{+}+2, n^{-}}\right. \\
& \left.\left.+\sqrt{\left(m^{-}\right)\left(n^{-}+1\right)\left(n^{-}+2\right)} A_{m^{+}, m^{-}-1, n^{+}, n^{-}+2}\right)\right]
\end{aligned}
$$

where the matrices $A$ and $B$ are related by a phase transformation

$$
A_{m^{ \pm}, n^{ \pm}}(x)=e^{-i\left(m^{+}+m^{-}+n^{+}+n^{-}+Z\right)(\gamma(x)-1) / x} B_{m^{ \pm}, n^{ \pm}}(x)
$$

with $\gamma(x)=2+Q x^{2}$ and $Z$ is an infinite constant (related to the renormalization of the vacuum energy). The dimensionless constant $\alpha$ has the value $\frac{\lambda V H}{8 \sqrt{2 \pi k \phi}}$. To arrive at equation (28), we have ignored terms that involve modes $\pm \frac{1}{2} \mathbf{k}$ and $\pm 4 \mathbf{k}$ since we are not concerned with how these modes evolve for our present purposes.

We begin the simulation for small values of $x$, well before the modes cross outside the Hubble length. At such a time, there has been a negligible amount particle production, so our initial wavefunction is simply the Fock vacuum, $|\psi\rangle_{i}=\left|m^{ \pm}=0\right\rangle\left|n^{ \pm}=0\right\rangle$. In the limit of $\frac{k}{a} \ll H$ or $x \ll 1$, this initial condition corresponds to the BunchDavies vacuum. During vacuum-energy-domination, the equation of state parameter, $w$, equals -1 . Therefore, neglecting the mass of the scalar field, the value of $Q$ is unity.

\section{B. Entanglement measures}

Discussions of decoherence rely on the notion of an environment: a collection of degrees of freedom that interacts and becomes entangled with the system of interest. Our model is naturally separated into modes with different magnitudes of momentum. Noting that the entanglement entropy does not depend on our choice of which set of modes is the environment and which is the system, we identify the modes with momentum $\pm 2 \mathbf{k}$ with the environmental degrees of freedom and the modes with momentum $\pm \mathbf{k}$ to be the system.

This choice represents an entanglement due to coarse graining the internal degrees of freedom of the scalar field based on scale. One can think of the coarse graining as either being due to practical limitations in the observations that can be made or as physical limitations such as a mode being entangled with a mode with a wavelength greater than the horizon size. The latter case is discussed in [18].
We measure the entanglement between modes using two different entanglement measures. The first of these is the entanglement or von Neumann entropy. The other is the linear entropy. While the former is more common, the latter is easier to compute and scales monotonically with the entanglement entropy. Figure 1 shows a comparison between these two measures for $\alpha=0.2$.

The density matrix of the above described system is

$$
\begin{aligned}
\rho & =|\psi\rangle\langle\psi| \\
& =\sum_{m^{ \pm}, n^{ \pm}, m^{\prime \pm}, n^{\prime \pm}=0}^{\infty} B_{m^{ \pm}, n^{ \pm}} B_{m^{\prime \pm}, n^{ \pm}}^{\dagger}\left|m^{ \pm}\right\rangle\left|n^{ \pm}\right\rangle\left\langle n^{\prime \pm}\right|\left\langle m^{\prime \pm}\right|
\end{aligned}
$$

and we assume that the modes with momentum $2 k$ are inaccessible to measurement. This gives rise to a reduced density matrix obtained from tracing over the unobserved degrees of freedom.

$$
\begin{aligned}
\rho_{N} & =\operatorname{Tr}_{M} \rho=\sum_{m^{\prime \prime \pm}=0}^{\infty}\left\langle m^{\prime \prime \pm} \mid \psi\right\rangle\left\langle\psi \mid m^{\prime \prime \pm}\right\rangle \\
& =\sum_{n^{ \pm}=0}^{\infty} \sum_{n^{ \pm}=0}^{\infty}\left(\sum_{m^{ \pm}=0}^{\infty} B_{m^{ \pm}, n^{ \pm}} B_{m^{ \pm}, n^{ \pm}}^{\dagger}\right)\left|n^{ \pm}\right\rangle\left\langle n^{\prime \pm}\right|
\end{aligned}
$$

The von Neumann entropy is then a measure of the entanglement between the $N$ system and the unobserved $M$ system.

$$
S=-\operatorname{Tr}\left(\rho_{N} \ln \rho_{N}\right)=-\sum_{i=1}^{N} \rho_{i} \ln \rho_{i}
$$

where the $\rho_{i}$ 's are the eigenvalues of the reduced density matrix, $\rho_{N}$. A system with a finite Hilbert space spanned 
by $N$ basis states will have a maximum entropy $S_{\max }=$ $\ln N$.

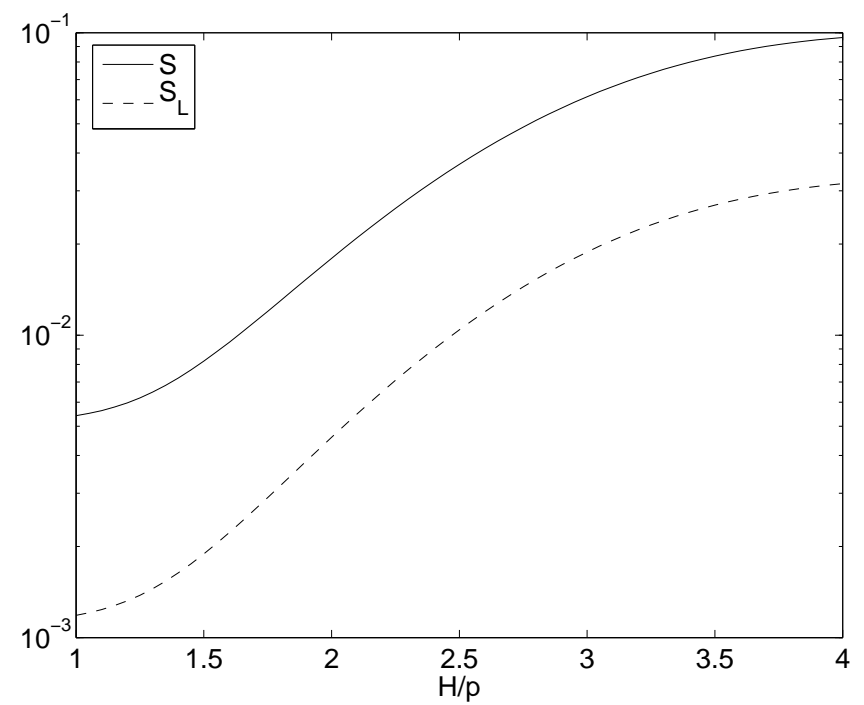

FIG. 1: The evolution of entanglement entropy, $S$, and linear entropy, $S_{L}=\left|1-\operatorname{Tr}\left(\rho^{2}\right)\right|$, as the mode stretches past the horizon for $\alpha=0.2$. This demonstrates that the nonlinearities in the inflaton potential are capable of producing entanglement entropy between the coupled modes. Also note that $S$ scales monotonically with $S_{L}$.

The linear entropy, $S_{L}=1-\operatorname{Tr}\left(\rho^{2}\right)$, is often used as a stand-in for the entanglement entropy since it can be computed more easily and in our case contains the same qualitative information,

$$
\operatorname{Tr}\left(\rho^{2}\right)=\sum_{i=1}^{\infty} \sum_{j=1}^{i} \begin{cases}2\left|\rho_{i j}\right|^{2} & \text { if } j \neq i \\ \left|\rho_{i j}\right|^{2} & \text { if } j=i .\end{cases}
$$

A system with a finite Hilbert space spanned by $N$ basis states will have a maximum linear entropy $S_{L, \max }=(N-$ $1) / N$.

From figure 1, we can see that this quantity is nearly proportional to the entropy. We will present the results both in terms of entanglement entropy and $S_{L}$.

\section{Thermal Entropy and Classicality}

The amount of entropy generated can be compared to the entropy of a thermal system that contains the same average number of particles. For a thermal system, the entropy is

$$
S_{\mathrm{th}}=-\sum_{n=1}^{\infty} \rho_{n, \mathrm{th}} \ln \rho_{n, \mathrm{th}}
$$

where the thermal density matrix is given by

$$
\rho_{n, \mathrm{th}}=\frac{e^{-\beta E_{n}}}{\sum_{n^{\prime}=1}^{\infty} e^{-\beta E_{n^{\prime}}}}
$$

and $n^{\prime}$ labels the Fock states. Since the energy is $m=$ $n^{+}+n^{-}$, each $n^{\prime}$ state is $m+1$ times degenerate, the partition function can be written

$$
\sum_{n^{\prime}=1}^{\infty} e^{-\beta E_{n^{\prime}}}=\sum_{m=0}^{\infty}(m+1) e^{-\beta m}=\frac{1}{\left(e^{-\beta}-1\right)^{2}} .
$$

Using the relation

$$
\langle n\rangle=\sum_{n^{\prime}=0}^{\infty} n \rho_{n^{\prime}, \text { th }}=\sum_{n=0}^{\infty} n(n+1) e^{-\beta n}\left(1-e^{-\beta}\right)^{2}
$$

we can eliminate $\beta$ for $\langle n\rangle$ using

$$
e^{-\beta}=\frac{\langle n\rangle}{2+\langle n\rangle}
$$

where $\langle n\rangle$ is the average number of particles in the reduced system. Finally, we can write the thermal entropy as

$$
S_{\mathrm{th}}(\langle n\rangle)=-\sum_{m=0}^{\infty}(m+1) \frac{4\langle n\rangle^{m}}{(2+\langle n\rangle)^{m+2}} \ln \left(\frac{4\langle n\rangle^{m}}{(2+\langle n\rangle)^{m+2}}\right)
$$

This quantity allows us to compare the entropy generated due to the coupling with the total energy of a thermal system at the same temperature. For example, if the information content of a system is defined as $I=S_{\mathrm{th}}-S$ then the relative information lost from the system due to the non-linear coupling term is

$$
I_{\mathrm{lost}}=1-\frac{I}{I_{\max }}=\frac{S}{S_{\mathrm{th}}} .
$$

Figure 2 shows that the rate of information loss due to the coupling is roughly the same as the rate of particle production.

Campo and Parentani argue that for Gaussian states at the threshold of separability and for $\langle n\rangle \gg 1$, the entanglement entropy between modes will be one half the entropy of the thermal state [13]. Since our states are not Gaussian, there is no known general separability condition. However, from the lack of growth in the information loss function shown in Figure 2, we can see that the Gaussian separability condition is unlikely to occur as $\langle n\rangle$ grows much larger than 1 at times greater than can be shown on the figure. Therefore, these types of non-linear interactions alone are likely insufficient to cause the system to appear classical.

Another measure of separability used by Campo and Parentani is the parameter $\delta$ defined by the equation

$$
|c|^{2}=n(n+1-\delta)
$$

where $n=\operatorname{Tr}\left(\rho a_{\mathbf{k}}^{\dagger} a_{\mathbf{k}}\right)=\left\langle n^{+}\right\rangle$and $c=\operatorname{Tr}\left(\rho a_{\mathbf{k}} a_{-\mathbf{k}}\right)$. The parameter, $\delta$, is a measure of the correlations between the $\mathbf{k}$ and $\mathbf{- k}$ modes. For Gaussian density matrices, it can be shown that separability occurs when $\delta=1$. The 


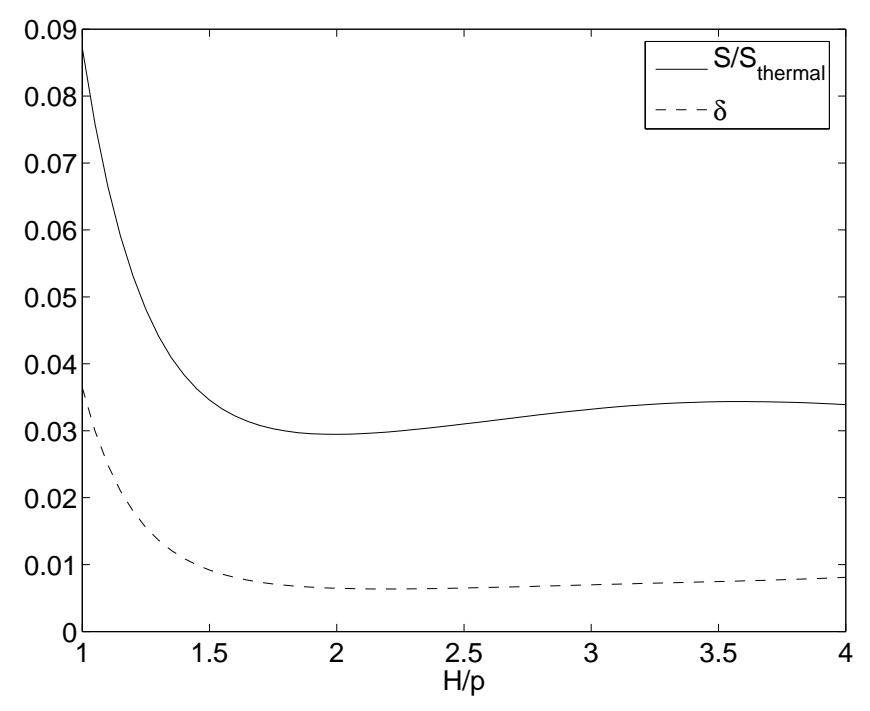

FIG. 2: The fraction of information lost due to tracing out the unobserved degrees of freedom, defined by equation (42), and the separability parameter $\delta$ (Eq. 43) as the observed mode stretches past the horizon for $\alpha=0.2$. By the end of the simulation, $I_{\text {lost }}$ appears to have leveled off to a constant few percent. For a separable (classical) system, we would expect $I_{\text {lost }}$ to grow to at least 0.5 to the right of the graph. If the system were to become classical, we would expect $\delta$ to grow to 1 . However, it too levels off to less than a percent.

value of $\delta$ measured for our model is shown alongside the information loss function in Figure 2 In both cases, the measures flatten out after the modes leave the horizon and fail to grow as one would need for non-linearities to explain the classicality of the quantum state. We can generalize the definition of $c$ to measure the correlation between modes of different magnitudes of momenta in our system

$$
d \equiv \operatorname{Tr}\left(\rho a_{-\mathbf{2} \mathbf{k}} a_{\mathbf{k}} a_{\mathbf{k}}\right)
$$

Although the interpretation of this quantity or $\delta$ is not as clear cut as for Gaussian density matrices, we find that both are useful and convenient tracers of the entanglement entropy.

\section{Estimating the sizes of $\lambda$ and $x_{\text {final }}$}

In order to match our above analysis with reality, we would like to make order of magnitude estimates for the parameters $\alpha$ in equation (18) and the final value of the $x$ at the end of inflation, $x_{\text {final }}$.

For fluctuations in a scalar field other than the inflaton, the value of $\lambda$ is essentially arbitrary; however, the gravitational self-interaction of the field provides a strict lower bound. Burgess, et al. 15] give an estimate of this self-interaction,

$$
\begin{aligned}
\lambda_{g} & \approx \frac{48}{(2 \epsilon)^{3 / 2}}\left(\frac{H}{M_{\mathrm{Pl}}}\right)^{2}=\frac{128 \pi}{(2 \epsilon)^{3 / 2}}\left(\frac{M}{M_{\mathrm{Pl}}}\right)^{4} \\
& \approx 6 \times 10^{-16}\left(\frac{\epsilon}{0.01}\right)^{-3 / 2}\left(\frac{M}{10^{14} \mathrm{GeV}}\right)^{4}
\end{aligned}
$$

where $M^{4}$ is the vacuum energy associated with the scalar field, and $\epsilon=\frac{M_{\mathrm{P} 1}^{2}}{2}\left(\frac{V^{\prime}}{V}\right)^{2}$ is a slow-roll parameter which may be larger than 1 if the scalar field is not the inflaton. We have included a possible matter-dominated period following the end of inflaton from scale factor $a_{\mathrm{EI}}$ to $a_{\mathrm{RH}}$ before reheating and taken $a$ to be the value of scale factor at the end of inflation.

The parameter $\alpha$ was introduced in equation (18) to replace

$$
\alpha=\frac{\lambda V H}{8 \sqrt{2 \pi k M_{\mathrm{Pl}}}}
$$

So, if we take, for example, a mode of size $\omega=c k \sim$ $0.1 \mathrm{~Hz}=5 \times 10^{-45} M_{\mathrm{Pl}}$ today, we arrive at an estimate for $\alpha$ due to gravitational self-interactions.

$$
\alpha_{g} \approx 2 \times 10^{-3}\left(\frac{\epsilon}{0.01}\right)^{-3 / 2}\left(\frac{M}{10^{14} \mathrm{GeV}}\right)^{6}\left(\frac{\omega}{0.1 \mathrm{~Hz}}\right)^{-1 / 2}
$$

If the scalar field in question is the inflaton field, the gravitational self-interaction will dominate over self-coupling interactions.

The analysis here has assumed that reheating is quick and efficient 222, 23], but in principle the end of inflaton may be followed by a period of matter domination from scale factor $a_{\mathrm{EI}}$ to $a_{\mathrm{RH}}$ before reheating. With this generalization, the comoving Hubble rate at the end of inflation is

$$
\begin{aligned}
a_{\mathrm{EI}} H & =\left(\frac{\pi^{2}}{30} g_{r} \frac{a_{\mathrm{EI}}}{a_{\mathrm{RH}}}\right)^{1 / 4}\left(\frac{8 \pi}{3}\right)^{1 / 2} \frac{T_{0} M}{M_{\mathrm{Pl}}} \\
& =6.3\left(g_{r} \frac{a_{\mathrm{EI}}}{a_{\mathrm{RH}}}\right)^{1 / 4} \frac{M}{10^{14} \mathrm{GeV}} \mathrm{MHz}
\end{aligned}
$$

where $M^{4}$ is the vacuum energy associated with the inflaton field, $\left(\approx \lambda M_{\mathrm{Pl}}^{4} / 4\right)$ and $g_{r}$ is the number of relativistic degrees of freedom at the end of reheating where the photon counts as two. The value of $x_{\text {final }}$ (at the end of inflation) for the comoving scale $a_{\mathrm{EI}} H$ is simply unity and for other scales we have

$$
x_{\text {final }}=\frac{a_{\mathrm{EI}} H}{\omega}=6.3 \times 10^{7} g_{r}^{1 / 4} \frac{M}{10^{14} \mathrm{GeV}} \frac{0.1 \mathrm{~Hz}}{\omega}
$$

Consequently although the correlations are present on all scales, they are most obvious on the comoving scale of the Hubble length at the end of inflation (i.e. really small scales). On these small scales the density fluctuations are well into the non-linear regime today but tensor fluctuations, gravitational waves $(\mathrm{GW})$, would still be a 
loyal tracer of these correlations. Inflationary tensor perturbations were first calculated in 24].

The expression given in Eq. 51 is very uncertain. Typically today's Hubble scale is assumed to pass out through the Hubble length during inflation about 50-60 $e$-foldings [7]; Eq. 51]gives $56 e$-foldings before the end, so the centihertz scale would pass through the Hubble length $12-22 e-$ foldings before the end of inflaton. However, the former number is highly uncertain. For example, if inflation occurs at a lower energy scale or if there is a epoch of late "thermal inflaton" [25, 26, 27], the number of $e$-foldings for today's Hubble scale could be as low as 25 [], yielding $x_{\text {final }} \ll 1$ for $c k \sim 0.1 \mathrm{~Hz}$.

Because the simulation increases in complexity as particles are produced (see figure 3), we are confined to keeping $x_{\text {final }} \sim O(1)$. So, even though $\alpha$ may be small in reality, there may be sufficient time during inflation for even a small non-linearity to produce a great deal of entanglement entropy because of very large values of $x_{\text {final }}$.

\section{RESULTS}

We would like to investigate how the amount of entropy generated in a single mode scales with the coupling strength and the duration of inflation (i.e. $\alpha$ and $x_{\text {final }}$ ). Figure 1 explicitly shows the creation of entanglement entropy for $\alpha=1$ as the universe undergoes its inflationary phase. The horizontal axis, $x=H / p$, is the physical size of a mode with respect to the horizon scale. The entanglement entropy increases less quickly than exponentially, which would be a straight line on the figure. Unfortunately, as was mentioned previously, the computational size of the problem prevents us from simulating far past horizon crossing because the number of particles becomes too large. Figure 3 shows how many Fock states are in the reduced system at each time step in the simulation. The number of states being integrated is this number to the $3 / 2$ power, and the number of entries in the density matrix is the square of this number.

The evolution of particles in the system is shown in figure 4 Our results are consistent with those found in Heyl 20] and show a nearly exponential evolution of the average particle number. Moreover, we can look at the evolution of each mode separately. For $\lambda=0$, each mode evolves according to the same equations of motion, and in this case, there is no difference between the rate that each of the modes evolves. However, the nature of the interaction between the modes is not symmetric because the decay of a single $M$ mode particle results in $2 \mathrm{~N}$ mode particles and therefore the interaction results in an increased rate of production of $N$ mode particles, relative to the $M$ mode. Figure 5 shows how the entanglement entropy scales with average particle number when $\alpha=$ 0.2 .

We performed the simulation for a variety of values for the coupling, $\alpha$, spanning several orders of magnitude. Figure $[$ shows entropy generation as a function of $\alpha$ for

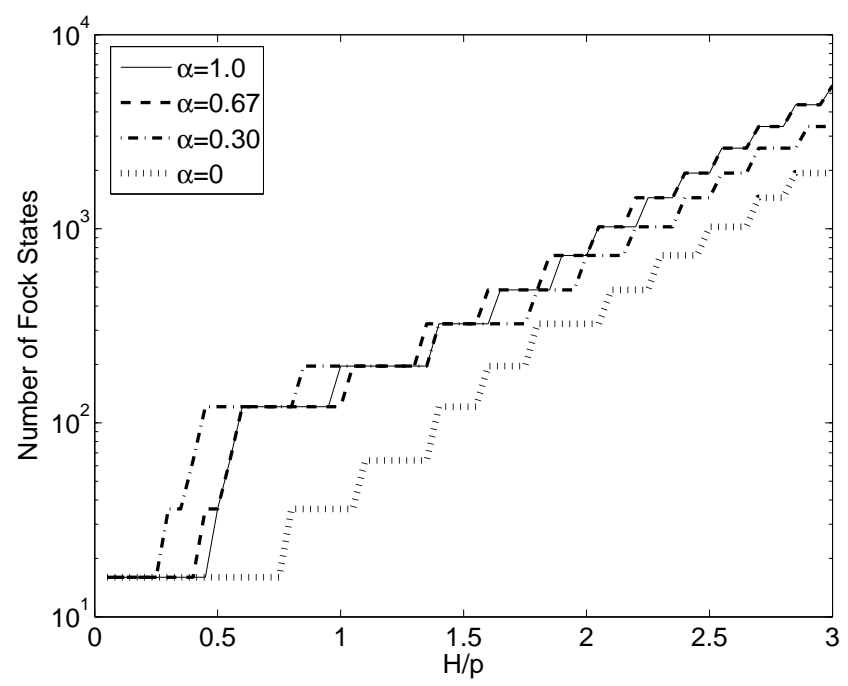

FIG. 3: The number of Fock states associated with the reduced system.

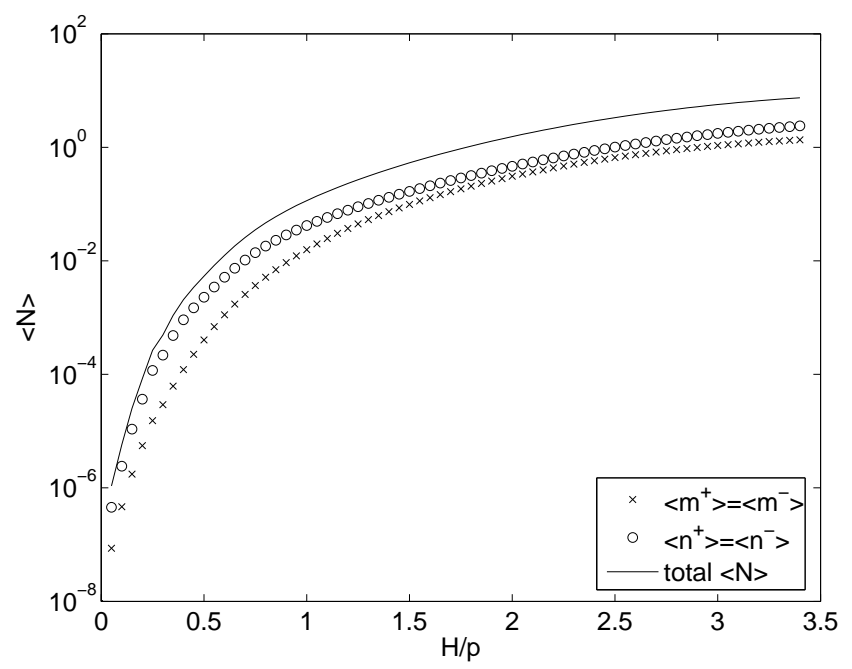

FIG. 4: Evolution of the average particle numbers for each mode for $\alpha=1.0$

a variety of inflation durations $x_{\text {final }}$. From this plot, we can see that $S_{\text {final }}$ scales roughly as a power law in $\alpha$. Most of the $\alpha$ dependence can be removed by dividing $S_{\text {final }}$ by $\alpha^{1.75}$. Doing this also helps to illustrate how $S_{\text {final }}$ scales with $x_{\text {final }}$. As expected, there is no entropy generated without the coupling terms (i.e. when $\alpha=0$ ). In this case, there is no communication between modes of the scalar field and they evolve independently.

As was mentioned earlier, $S_{L}$ is a useful stand-in for $S$ that can be computed faster than $S$. Figure 8 echoes the previous results in terms of $S_{L}$ instead of $S$. In this case, $1-\operatorname{Tr}\left(\rho^{2}\right)$ scales more like $\alpha^{2}$ instead of $\alpha^{1.75}$. However, both $S_{L}$ and $S$ demonstrate the same qualitative behaviour.

In addition, we have found other useful stand-ins for the entanglement entropy that are easier to compute and 


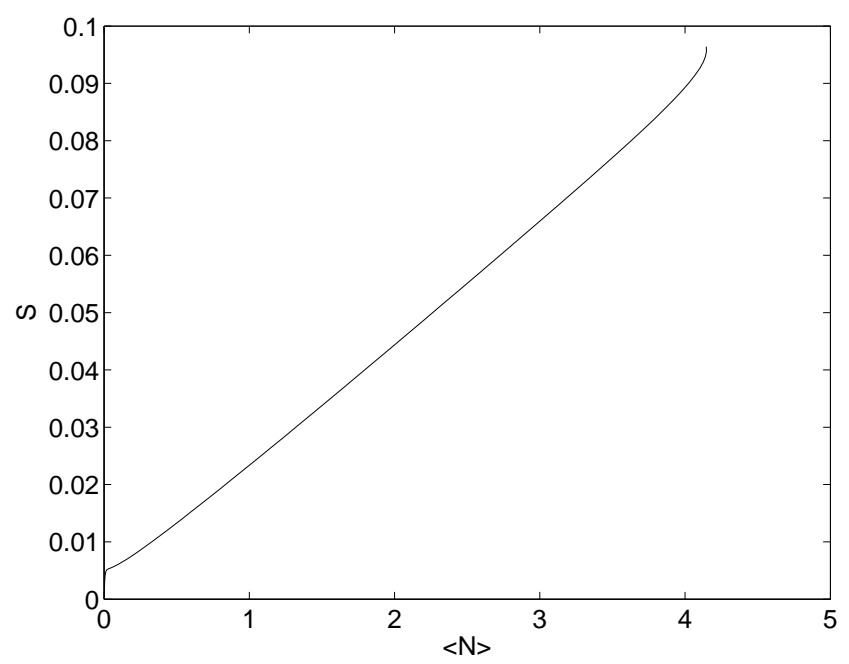

FIG. 5: Entanglement entropy vs. average particle number for $\alpha=0.2$

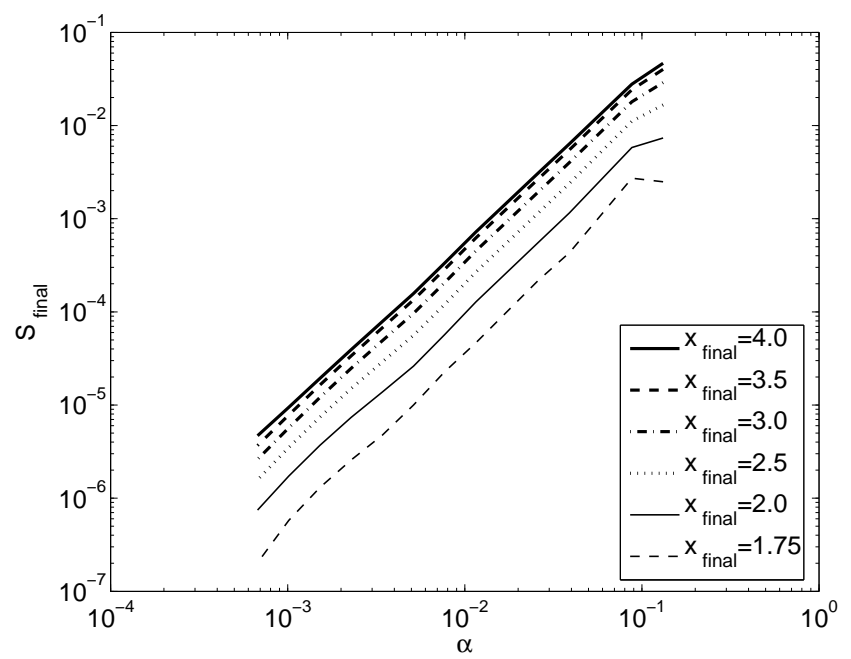

FIG. 6: von Neumann entropy vs. $\alpha$ for various values of $x_{\text {final }}$

scale similarly with $\alpha$. The $\delta$ parameter, defined in (43), scales roughly like an $\alpha^{2}$ power law much like $S_{L}$. Figure 9 shows the power law behaviour of this function. Additionally, if we use a simple measure of correlation between entangled modes, $|d|^{2}$ (Eq. 44), we find that its scales like $\alpha^{1.85}$ (see fig. 7), and so can be a useful stand-in for the von Neumann entropy, $S \propto \alpha^{1.75}$.

In the real universe, we are dealing with small values of $\lambda$ and very large values of $x$. However, the simulation outlined in this paper is limited because its computational complexity increases dramatically as particles are produced, even for small values of the coupling, $\alpha$. Moreover, for small values of $\alpha$, the production of entropy is too small to be meaningful. While the dependence of $S$ on $\alpha$ nearly follows a power law, there is no simple relation describing the dependence of $S$ on $x_{\text {final }}$. The value

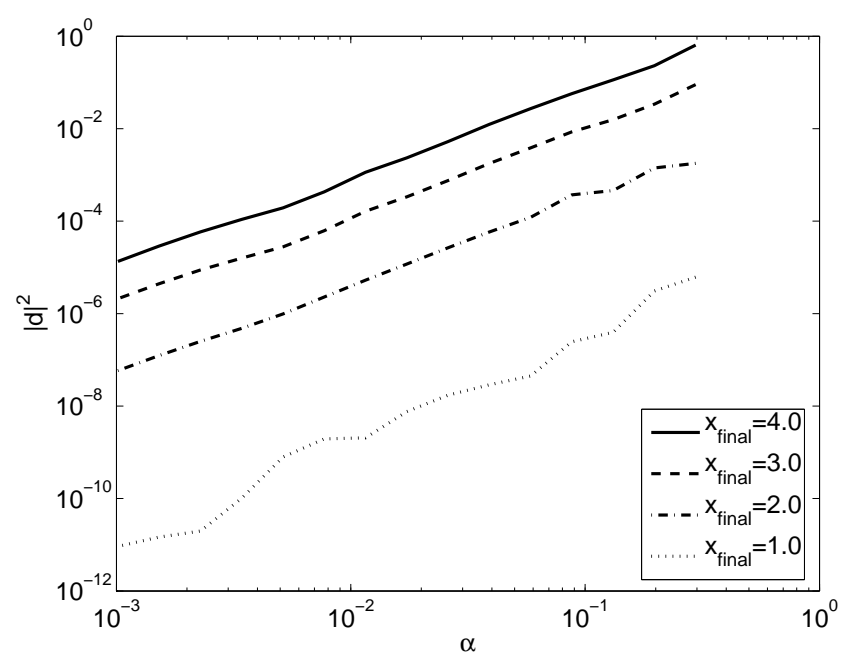

FIG. 7: $|d|^{2}=\left|\operatorname{Tr}\left(\rho a_{\mathbf{2} \mathbf{k}} a_{\mathbf{k}} a_{\mathbf{k}}\right)\right|^{2}$ scales with $\alpha$ in much the same way as $S$, but is less costly to compute.

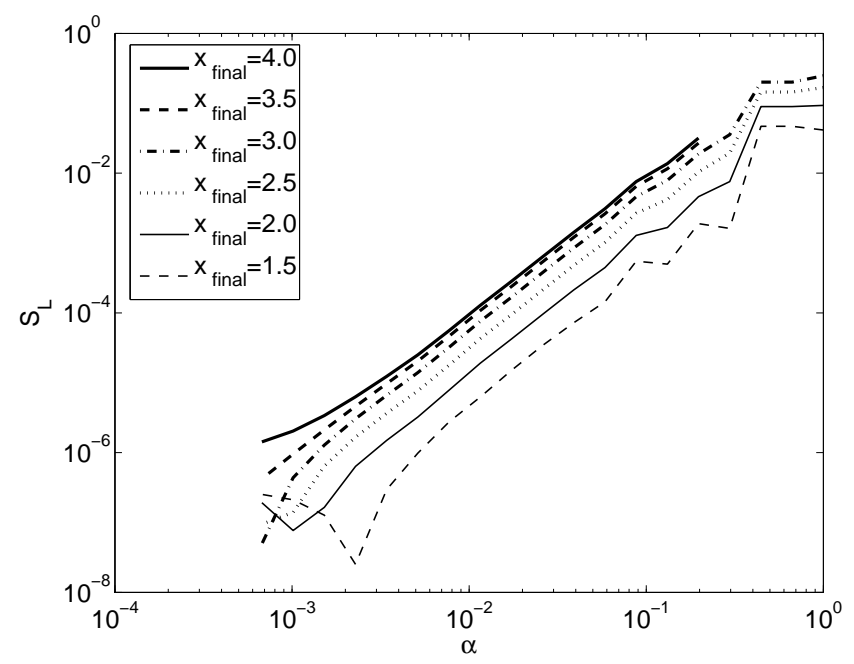

FIG. 8: $S_{L}$ vs. $\alpha$ for various values of $x_{\text {final }}$

$S_{L}$ is approximately proportional to $\alpha^{2} x^{3}$ over a wide range of $\alpha$ and the modest range $x$ probed by the simulations; therefore, very roughly, we can write the scaling law as $S_{L} \propto \alpha^{2} x_{\text {final }}^{3}$ where

$$
S_{L} \approx 10^{16} g_{r}^{3 / 4}\left(\frac{M}{10^{14} \mathrm{GeV}}\right)^{15}\left(\frac{\omega}{0.1 \mathrm{~Hz}}\right)^{-4} .
$$

Of course, only values of $S_{L}$ less than unity make sense, so a larger value from the fitting formula indicates that $S_{L}$ is very close to one. However, a value of $S_{L}<1$ is obtained by lowering the mass scale of inflation below

$$
M<8 \times 10^{12}\left(\frac{\omega}{0.1 \mathrm{~Hz}}\right)^{-4 / 15} \mathrm{GeV}
$$

therefore, if the energy scale of inflation is low, the quantum states of fluctuations at $\omega \sim 0.1 \mathrm{~Hz}$ will remain coherent despite the non-linear coupling. 


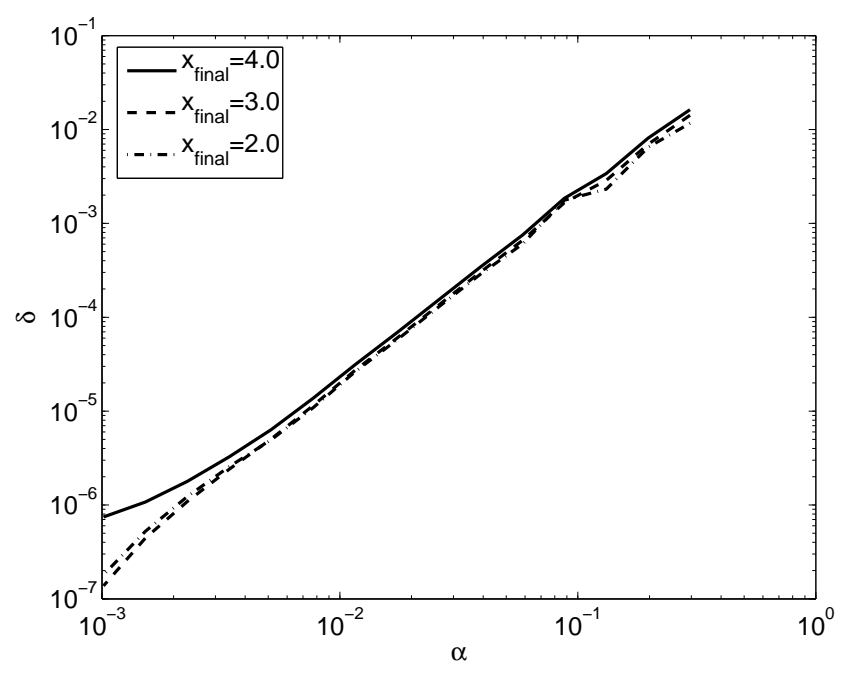

FIG. 9: $\delta$, defined in equation (43) scales with $\alpha$ in much the same way as $S_{L}$, but is less costly to compute.

The simulation was checked for consistency in several ways. First, we traced the probability throughout the simulation measured both by the sum of squares of the matrix elements $\sum_{m^{ \pm}, n^{ \pm}=0}^{\infty} A_{m^{ \pm}, n^{ \pm}}$and the trace of the density operator. Both of these quantities were conserved to a few parts in $10^{-7}$. Moreover, we estimated the level of numerical error by rerunning the simulation with a variety of phase rotations multiplying the initial wavefunction. The standard deviation of the results from these numerical changes in the initial conditions give us an idea of the level of numerical error in the simulation, which were typically at the level of one part per thousand.

\section{CONCLUSIONS}

In this paper we have developed a model in which two modes of a scalar field evolve during inflation and we have computed the entanglement entropy between them. The entanglement entropy generated between observed and unobserved modes in the inflaton field give the appearance that entropy is being produced, even though the scalar field remains in an overall pure state. The preceding results clearly show that non-linearities in the inflaton potential give rise to a generation of entanglement entropy between observed modes and unobserved modes in a scalar field during inflation. This entropy is an additional source to that caused by coupling to ex- ternal degrees of freedom [28], entanglement between the inside and outside of the horizon [29] and that which is created during reheating after inflation has ended.

We have attempted to extrapolate the results of our simulation to the real universe. The relevant parameters determining the amount of entropy generated via nonlinearities are the strength of the coupling $\alpha_{g} \sim 10^{-3}$ and the scale of the fluctuation at the end of inflation given by the dimensionless parameter $x_{\text {final }} \sim 10^{7}$. The entanglement entropy was found to scale like $\alpha^{1.75}$ for a fixed $x_{\text {final }}$. The dependence of $S_{L}$ on $x_{\text {final }}$ for a given value of $\alpha$ is not as straightforward, but $S_{L} \propto x_{\text {final }}^{3}$ over a short range of $x_{\text {final }}$ values. Based on these rough scaling patterns, we estimate that non-linearities due to gravity and inflaton self-coupling are insufficient to decohere modes that spend only a few Hubble times at super-horizon scales. In particular, if the energy scale of inflaton is less than $10^{13} \mathrm{GeV}$, fluctuations at about $0.1 \mathrm{~Hz}$ may remain coherent.

We found two measures of the decoherence related to the correlations between modes of different momenta provide a faithful estimate of the entanglement entropy in our model - one of these measures is new to this work (d) and specifically probes the non-linear coupling between modes. In particular these estimates are very inexpensive to calculate as compared to the von Neumann entropy and should prove useful for more detailed models of entropy generation.

It is usually assumed that the main contribution to the entropy observed in the density perturbations is generated during reheating, when the inflaton decays. However, the analysis demonstrates that entropy can be generated independently of reheating provided there is even a small non-linearity in the scalar potential; therefore, the results are applicable to scalar fields that do not participate in reheating. For example, the gravitational wave background can be treated as a pair of scalar fields, so even tensor fluctuations may contribute to the entropy and the classicality of the distribution of density perturbations in this way and observations of the gravitational wave background at high frequency could reveal the quantum mechanical origin of density fluctuations.

Acknowledgments This research was supported by funding from NSERC. The calculations were performed on computing infrastructure purchased with funds from the Canadian Foundation for Innovation and the British Columbia Knowledge Development Fund.
[1] A. H. Guth. Inflationary universe: A possible solution to the horizon and flatness problems. Phys. Rev. D, 23:347-356, January 1981.

[2] A. H. Guth and S.-Y. Pi. Fluctuations in the new inflationary universe. Physical Review Letters, 49:1110-1113,
October 1982.

[3] A. A. Starobinsky. Dynamics of phase transition in the new inflationary universe scenario and generation of perturbations. Physics Letters B, 117:175-178, November 1982. 
[4] D. H. Lyth. Large-scale energy-density perturbations and inflation. Phys. Rev. D , 31:1792-1798, April 1985.

[5] V. F. Mukhanov and G. V. Chibisov. Quantum fluctuations and a nonsingular universe. Soviet Journal of Experimental and Theoretical Physics Letters, 33:532, May 1981.

[6] S.W. Hawking. The development of irregularities in a single bubble inflationary universe. Phys. Lett. B, 115:295297, 1982.

[7] A.R. Liddle and D. H. Lyth. Cosmological inflation and large-scale structure. Cambridge University Press, Cambridge, U.K., 2000.

[8] C. Kiefer and D. Polarski. Why do cosmological perturbations look classical to us? Adv. Sci. Lett., 2:164-173, 2009.

[9] C. Kiefer, D. Polarski, and A. A. Starobinsky. Entropy of gravitons produced in the early universe. Phys. Rev. D , 62(4):043518, August 2000.

[10] D. Campo and R. Parentani. Inflationary spectra and partially decohered distributions. Phys. Rev. D, 72(4):045015, August 2005.

[11] D. Campo and R. Parentani. Inflationary spectra and violations of Bell inequalities. Phys. Rev. D , 74(2):025001, July 2006.

[12] T. Prokopec and G. I. Rigopoulos. Decoherence from isocurvature perturbations in inflation. Journal of Cosmology and Astro-Particle Physics, 11:29, November 2007.

[13] David Campo and Renaud Parentani. Decoherence and entropy of primordial fluctuations. i. formalism and interpretation. Physical Review D (Particles, Fields, Gravitation, and Cosmology), 78(6):065044, 2008.

[14] D. Campo and R. Parentani. Decoherence and entropy of primordial fluctuations. II. The entropy budget. Phys. Rev. D , 78(6):065045, September 2008.

[15] Cliff P. Burgess, R. Holman, and D. Hoover. On the decoherence of primordial fluctuations during inflation. 2006.

[16] Wojciech H. Zurek, Salman Habib, and Juan Pablo Paz. Coherent states via decoherence. Phys. Rev. Lett., 70(9):1187-1190, Mar 1993.
[17] Fernando Lombardo and Francisco D. Mazzitelli. Coarse graining and decoherence in quantum field theory. Phys. Rev., D53:2001-2011, 1996.

[18] Patrick Martineau. On the decoherence of primordial fluctuations during inflation. Class. Quant. Grav., 24:5817-5834, 2007.

[19] Viatcheslav F. Mukhanov, H. A. Feldman, and Robert H. Brandenberger. Theory of cosmological perturbations. Phys. Rept., 215:203-333, 1992.

[20] Jeremy S. Heyl. Quantum mechanical fluctuations at the end of inflation. Journ Phys A, 40:13997-14010, 2007.

[21] C. Armendariz-Picon. Why should primordial perturbations be in a vacuum state? Journal of Cosmology and Astro-Particle Physics, 2:31, February 2007.

[22] L. Kofman, A. Linde, and A. A. Starobinsky. Towards the theory of reheating after inflation. Phys. Rev. D, 56:3258-3295, September 1997.

[23] J. P. Zibin. Long wavelength cosmological perturbations and preheating. $\mathrm{PhD}$ thesis, Unversity of British Columbia, 2004.

[24] A. A. Starobinsky. Spectrum of relic gravitational radiation and the early state of the universe. JETP Lett., 30:682, 1979 .

[25] D. H. Lyth and E. D. Stewart. Thermal inflation and the moduli problem. Phys. Rev. D , 53:1784-1798, February 1996.

[26] M. Dine, L. Randall, and S. Thomas. Supersymmetry Breaking in the Early Universe. Physical Review Letters, 75:398-401, July 1995.

[27] M. Dine, L. Randall, and S. Thomas. Baryogenesis from flat directions of the supersymmetric standard model. Nuclear Physics B, 458:291-323, February 1996.

[28] Claus Kiefer, Ingo Lohmar, David Polarski, and Alexei A. Starobinsky. Pointer states for primordial fluctuations in inflationary cosmology. Class. Quant. Grav., 24:16991718, 2007.

[29] Jonathan W. Sharman and Guy D. Moore. Decoherence due to the horizon after inflation. J. Cosmol. Astropart. Phys., 2007. 